\title{
Three-port mediastino-laparoscopic esophagectomy (TPMLE) for an 81-year-old female with early-staged esophageal cancer: a case report of combining single-port mediastinoscopic esophagectomy and reduced port laparoscopic surgery
}

\author{
Di Lu ${ }^{1 \#}$, Xiguang Liu ${ }^{1 \#}$, Mei Li ${ }^{1 \#}$, Siyang Feng ${ }^{1 \#}$, Xiaoying Dong ${ }^{1}$, Xuezhou Yu ${ }^{2}$, Hua Wu ${ }^{1}$, Gang Xiong ${ }^{1}$, \\ Ruijun Cai ${ }^{1}$, Guoxin $\mathrm{Li}^{3}$, Kaican $\mathrm{Cai}^{1}$ \\ ${ }^{1}$ Department of Thoracic Surgery, Nanfang Hospital, Southern Medical University, Guangzhou 510515, China; ${ }^{2}$ Department of Thoracic Surgery, \\ Pengpai Memorial Hospital, Shanwei 516600, China; ${ }^{3}$ Department of General Surgery, Nanfang Hospital, Southern Medical University, Guangzhou \\ 510515, China \\ \#These authors contributed equally to this work. \\ Correspondence to: Kaican Cai. Department of thoracic surgery, Nanfang Hospital, Southern Medical University, No. 1838 of North Guangzhou \\ Avenue, Guangzhou 510515, Guangdong, China. Email: doc_cai@163.com.
}

Submitted Jan 05, 2018. Accepted for publication Apr 25, 2018.

doi: $10.21037 /$ jtd.2018.05.52

View this article at: http://dx.doi.org/10.21037/jtd.2018.05.52

\section{Introduction}

Esophageal cancer is the eleventh most common cancer worldwide, and the sixth most common cause of cancer related mortality due to its poor prognosis (1). Surgery remains the best option for operable patients with esophageal carcinoma. However, due to its anatomical property, radical resection is highly invasive and traumatic, and may cause severe complications and even death.

To lower the incidence of perioperative complications and mortality, minimally invasive approaches such as videoassisted thoracic surgery (VATS) have been developed for esophagectomy. However, single lung ventilation is still needed for VATS esophagectomy and lung injury cannot be avoided. For this reason, Fujiwara et al. developed an en bloc lymphadenectomy method in the upper mediastinum with a single-port mediastinoscopic (SPM) cervical approach $(2,3)$ to avoid single lung ventilation and potential lung injury. This method has provided a new option for patients with poor cardiopulmonary function or extensive pleural adhesion.

For the abdominal portion of esophagectomy, gastric mobilization with lymphadenectomy is usually performed laparoscopically with five small incisions for trocars in the conventional way, while reduced port laparoscopic surgery (RPLS) has been recognized as a less invasive way alternative to conventional laparoscopic surgery for patients with gastric cancer $(4,5)$; in these cases, only two small incisions should be made.

To make esophagectomy less invasive than before, we combined these two newly developed approaches together in an 81-year-old patient and named it three-port mediastino-laparoscopic esophagectomy (TPMLE). To the best of our knowledge, no one has reported the combined application of both SPM esophagectomy (SPME) and RPLS for patients with esophageal cancer.

\section{Case presentation}

A rough lesion of the esophageal mucosa, 23 centimeters from the incisors, was found by annual gastroscopic examination in an 81-year-old Chinese female, and was pathologically diagnosed as esophageal squamous cell cancer (ESCC) by biopsy. The patient presented without dysphagia and had no history of chronic disease. Endoscopic esophageal ultrasonography revealed tumor invasion to be superficial to the muscularis propria with one enlarged lymph node along the left upper esophagus. Positron emission tomography-computed tomography 
A

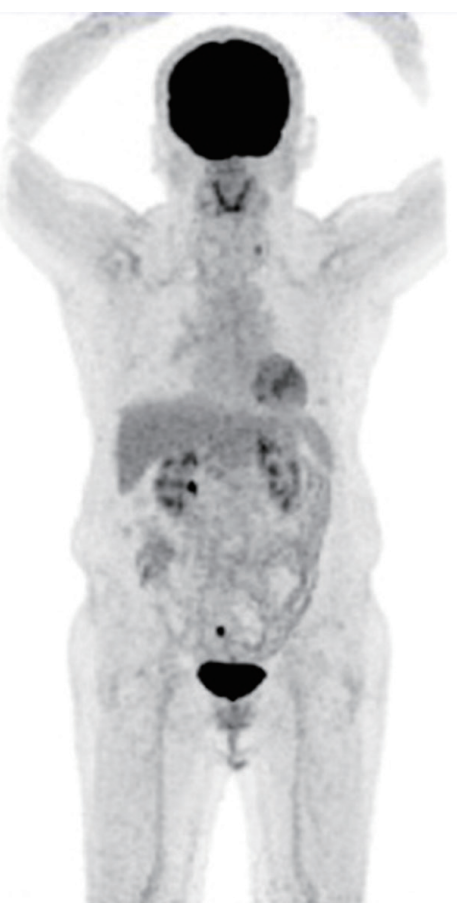

B
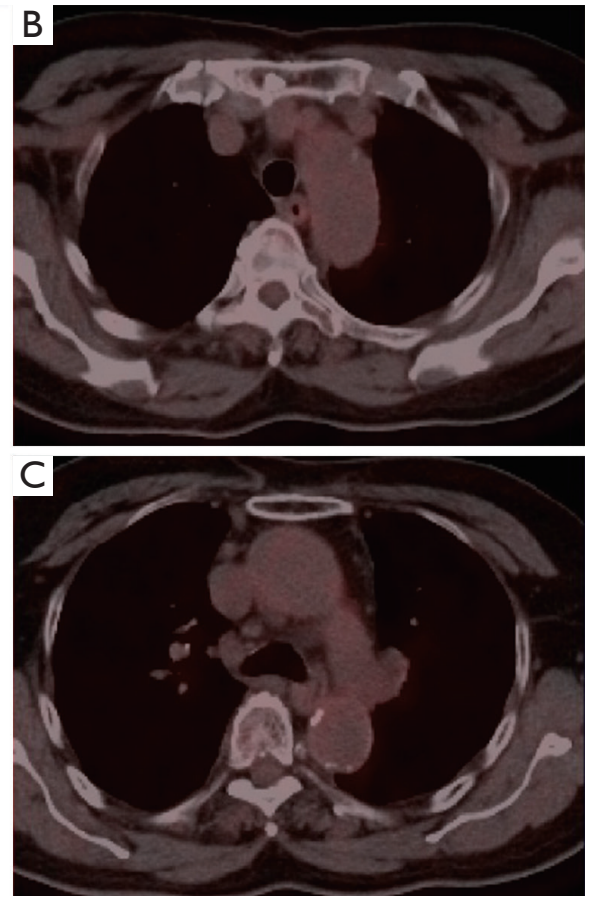

Figure 1 Preoperative PET-CT findings. (A) PET-CT shows there's no metastasis of other distant organs except the esophagus; (B) PETCT shows there's a slightly hyper metabolic lesion of the esophagus at the level of aortic arch; (C) PET-CT shows there's a slightly hyper metabolic lesion of the esophagus at the level of the tracheal bifurcation. PET-CT, positron emission tomography-computed tomography.

(PET-CT) scan suggested a slightly hypermetabolic mass in the upper esophagus without metastasis to distant organs (Figure 1). The patient was staged as cT1N1M0 (stage I) before surgery according to the $8^{\text {th }}$ staging system by AJCC/ UICC (6).

After group discussion with general surgery, anesthesiology, oncology and digestive disease departmental experts, radical resection was selected as the best way to treat this patient. Esophagectomy and mediastinal and cervical lymphadenectomy could be performed through the SPM approach to avoid single lung ventilation and potential lung injury. Reduced port laparoscopic gastrectomy with D2 lymphadenectomy has been routinely performed on gastric cancer patients in our hospital by general surgeons, but had not been applied for stomach mobilization and lymphadenectomy in patients with esophageal cancer. After further discussion and evaluation, a combination of the SPM approach and reduced port laparoscopic technique was decided, with the stomach portion to be performed intraperitoneally instead of the conventional method of pulling the stomach through an upper abdominal middle incision, to decrease the injury as much as possible.
The operation was performed as planned, with one group of thoracic surgeons performing SPM esophagectomy at the head, and another group of general surgeons performed the reduced port laparoscopic stomach mobilization and lymphadenectomy at the foot (Figure 2). In total, only three minimally invasive incisions (one left collar incision, one umbilicus incision and one right upper quadrant incision) were made (Figure 3). The duration of the whole operation was 203 minutes and 20 lymph nodes were dissected. Final pathological examination suggested moderately differentiated ESCC invading the submucosa and superficial to the muscularis propria, with one lymph node metastasis along the left recurrent laryngeal nerve (LRLN), which was staged as pT1bN1M0G2 ESCC, stage IIB (7). The patient recovered rapidly with mild incisional pain and began oral feeding on postoperative day 3 . At the one-month follow-up visit, there were no complications and her self-care ability had returned.

\section{Discussion}

For decades, thoracic surgeons have been developing 

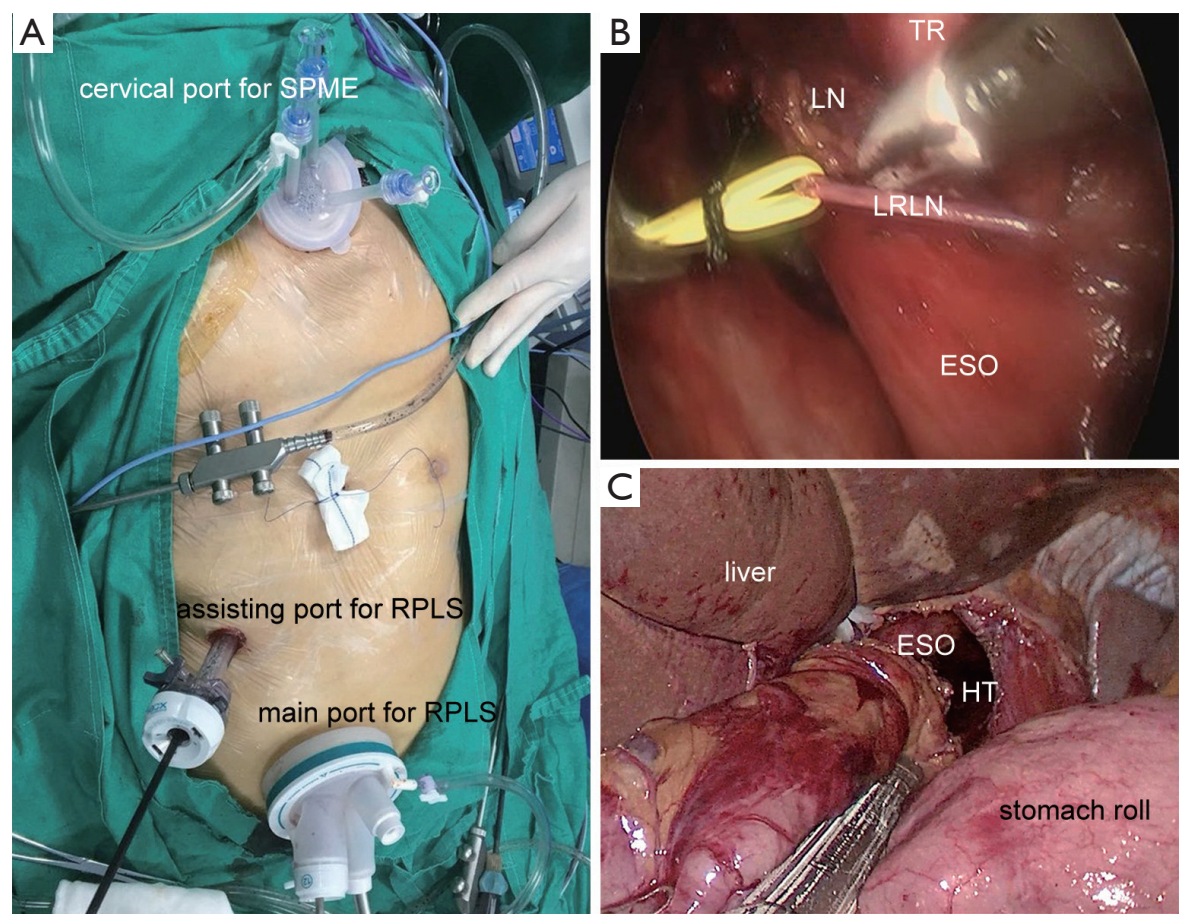

Figure 2 Intraoperative picture of the case undergoing TPMLE. (A) Three ports were used in the operation: cervical port for SPME, abdominal port and main port for RPLS; (B) LRLN was skeletonized and the LN along it was dissected; (C) the stomach roll was made intraperitoneally. TPMLE, three-port mediastino-laparoscopic esophagectomy; SPME, single-port mediastinoscopic esophagectomy; RPLS, reduced port laparoscopic surgery; ESO, esophagus; TR, trachea; LRLN, left recurrent laryngeal nerve; LN, lymph node; HT, hiatus.

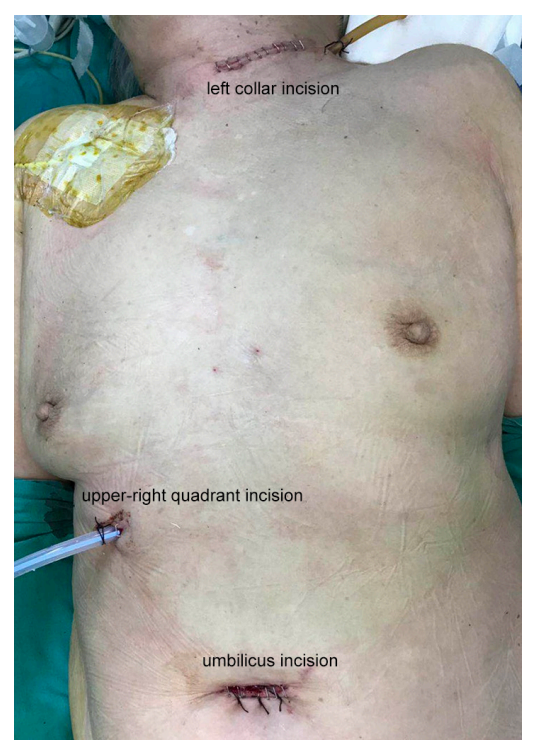

Figure 3 Three incisions were made for the operation: one left collar incision, one umbilicus incision and an upper-right quadrant incision. less invasive approaches for esophagectomy due to the complicated anatomic properties of the esophagus. It has been shown by a multicenter open-label randomized control trial that there is a lower incidence of postoperative short-term pulmonary infections, shorter hospitalstay, and better short-term quality of life in patients undergoing minimally invasive esophagectomy (MIE) versus open esophagectomy (8). However, for high-risk patients with comorbidities, or those who cannot tolerate one-lung ventilation, transthoracic MIE also appears to be too invasive. The mediastinal approach would be another option as one-lung ventilation is not required. Before Fujiwara reported his SPM approach of en bloc lymphadenectomy method in the upper mediastinum and esophagectomy, the mediastinal approach has always been denounced for its insufficient mediastinal lymphadenectomy $(9,10)$. According to Fujiwara's approach, lymphadenectomy along the left recurrent laryngeal nerve and under the aortic arch is much easier 
compared with the transthoracic approach, which is the most difficult.

On the other hand, Fujiwara's method does not recommend mediastinal and abdominal procedures to be performed simultaneously, because the posterior space of the lymph nodes along the tracheal bifurcation needs to be exposed through the cervical approach first; then, the anterior plane of these lymph nodes can be easily separated by the transhiatal procedure $(2,3)$. According to our experience and the case presented above, two groups of surgeons could perform the two parts simultaneously. The general surgeons can wait for the thoracic surgeons to expose the posterior space of the lymph nodes along the tracheal bifurcation and the azygos arch first for further lymphadenectomy, which saves anesthesia time. In this way, if the high-risk patients cannot tolerate a long period of anesthesia, the two parts can be performed at the same time with the two groups of surgeons saving time by working in coordination for en bloc lymphadenectomy.

In addition, though the rapid recovery of this patient could be due to the avoidance of single lung ventilation, the reduced number of incisions might also have contributed her recovery, compared with Fujiwara's approach. His new method of en bloc lymphadenectomy in the upper mediastinum and esophagectomy with a SPM cervical approach has drawn much attention worldwide, but should be further improved and evaluated for efficiency and safety before drawing a final conclusion. Attempting to perform less invasive operations on patients with esophageal cancer is an ongoing process.

In conclusion, we report for the first time, a case that combined application of SPM cervical approach and RPLS for a high-risk patient with early-staged esophageal cancer.

\section{Acknowledgements}

Funding: The study was supported by the Science and Technology Planning Project of Guangdong Province (2014A020212541) and Presidential Foundation of Nanfang Hospital, Southern Medical University (2016B018).

\section{Footnote}

Conflicts of Interest: The authors have no conflicts of interest to declare.

Informed Consent: We have obtained the patient's signed consent form for publication of all the details of her case in journals without showing her name and face, which is available from the corresponding author on reasonable request.

\section{References}

1. Global Burden of Disease Cancer Collaboration, Fitzmaurice C, Allen C, et al. Global, Regional, and National Cancer Incidence, Mortality, Years of Life Lost, Years Lived With Disability, and Disability-Adjusted Lifeyears for 32 Cancer Groups, 1990 to 2015: a systematic analysis for the global burden of disease study. JAMA Oncol 2017;3:524-48.

2. Fujiwara H, Shiozaki A, Konishi H, et al. Singleport mediastinoscopic lymphadenectomy along the left recurrent laryngeal nerve. Ann Thorac Surg 2015;100:1115-7.

3. Fujiwara H, Shiozaki A, Konishi H, et al. Perioperative outcomes of single-port mediastinoscope-assisted transhiatal esophagectomy for thoracic esophageal cancer. Dis Esophagus 2017;30:1-8.

4. Li GX, Zhang C, Yu J, et al. A new order of D2 lymphadenectomy in laparoscopic gastrectomy for cancer: live anatomy-based dissection. Minim Invasive Ther Allied Technol 2010;19:355-63.

5. Lin T, Mou TY, Hu YF, et al. Reduced Port Laparoscopic Distal Gastrectomy with D2 Lymphadenectomy. Ann Surg Oncol 2018;25:246.

6. Rice TW, Ishwaran H, Blackstone EH, et al. Recommendations for clinical staging (cTNM) of cancer of the esophagus and esophagogastric junction for the 8th edition AJCC/UICC staging manuals. Dis Esophagus 2016;29:913-9.

7. Rice TW, Ishwaran H, Hofstetter WL, et al. Recommendations for pathologic staging (pTNM) of cancer of the esophagus and esophagogastric junction for the 8th edition AJCC/UICC staging manuals. Dis Esophagus 2016;29:897-905.

8. Biere SS, van Berge Henegouwen MI, Maas KW, et al. Minimally invasive versus open oesophagectomy for patients with oesophageal cancer: a multicentre, open-label, randomised controlled trial. Lancet 2012;379:1887-92. 
9. Ikeda Y, Niimi M, Kan S, et al. Mediastinoscopic esophagectomy using carbon dioxide insufflation via the neck approach. Surgery 2001;129:504-6.

10. Parker M, Bowers SP, Goldberg RF, et al. Transcervical

Cite this article as: Lu D, Liu X, Li M, Feng S, Dong X, Yu X, Wu H, Xiong G, Cai R, Li G, Cai K. Three-port mediastinolaparoscopic esophagectomy (TPMLE) for an 81-year-old female with early-staged esophageal cancer: a case report of combining single-port mediastinoscopic esophagectomy and reduced port laparoscopic surgery. J Thorac Dis 2018;10(5):E378E382. doi: $10.21037 /$ jtd.2018.05.52 videoscopic esophageal dissection during two-field minimally invasive esophagectomy: early patient experience. Surg Endosc 2011;25:3865-9. 